\title{
Experimental Research on Flight Patterns of Bees
}

\author{
H.Y. Zhao, P.F. Zhang, Y. Ma, J.G. Ning \\ Beijing Institute of Technology
}

\begin{abstract}
For the research on the bees flight, the experimental platform combined of smoke wind tunnel and high speed camera has been set up. The information of bees flight on the plane can be synchronously recorded by two high speed cameras. By the analysis of pixel coordinates of bees wings on the photographs, the changing rules of wings in the space can be obtained with the establishment of bees space coordinates system. As a result, the changing rules of position angle and stroke angle can also be obtained. With the analysis, we have found that the duration of up-flapping is different with that of down-flapping. During the terminal stage, the wings accelerate quickly. During the period when down-flapping turns into upflapping, the wings rotation takes part of the duration. And during the rotation, the stroke angle scarcely changes.
\end{abstract}

\section{Keywords-honeybee; stroke angle; position angle}

\section{INTRODUCTION}

There are many advantages in bees flying. And plenty of aerodynamicists have been focusing on the bees flight. In the early 1960s, Weis-fogh[1] began the research on the bees flight. Sane and Dickison[2] [3] studied the aerodynamic characteristics of flapping flight with mechanical bionic flapping wings. Birch and Dickison [4] studied the wake effect of flapping flight by the use of mechanical flapping wings. Ellington[5] [6] summed up insects flight rules including insects body feature and wings track during flight by one high speed camera. The calculation method of Ellington by use of only one high speed camera is complicated and too many restricting factors affect the calculation results. In this paper, two high speed cameras with fixed angle have been used. The wings track is calculated by projection relationship of straight line and plane. Compared with other insects, bees have advantage in small volume, flexible motion and easy to catch. So, bees are chosen as the experimental research object. And to capture the bees wings track and get the meticulous changing rules of bees flapping, the bees are belayed.

\section{EXPERIMENTAL PLATFORM}

The experimental platform is shown in Fig. 1. The frame rate of the high speed camera 1 and 2 is $10000 \mathrm{fps}$. The exposure time is $90 \mu \mathrm{s}$. The picture resolution is $528 \times 400$ pixel. Additional extension tube with $85 \mathrm{~mm}$ camera lens is used in high speed camera. Two high speed cameras are used for the experiment. One is fixed right above the wind tunnel and another is fixed right ahead of the wind tunnel. Two LED lights placed in diagonal direction of the wind tunnel experimental section are optical source.

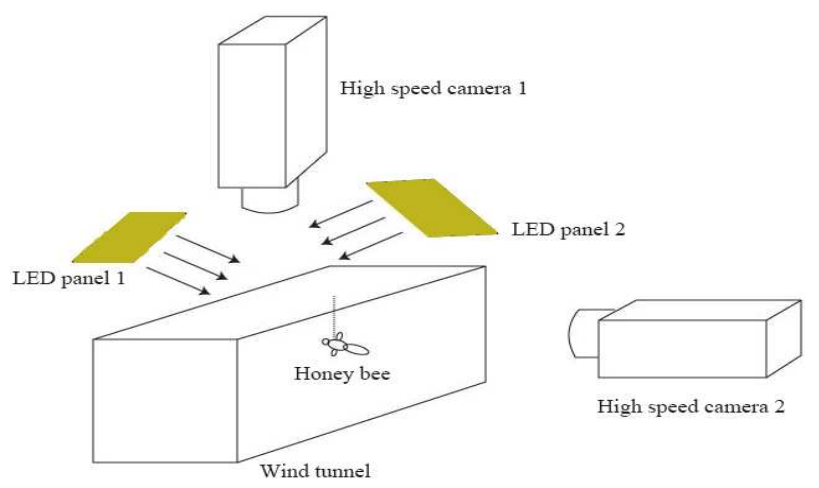

FIGURE I. PHOTOGRAPH OF THE EXPERIMENTAL PLATFORM.

\section{PROJECTION RELATIONSHIP OF STRAIGHT LiNE AND PLANE}

During the bees flying, two high speed cameras that separately film plane A and plane B record the information synchronously, as shown in Fig. 2. High speed camera 1 films the horizontal plane, plane A, from the top of the wind tunnel. And high speed camera 2 films the plane B perpendicular to plane A, from the front of the wind tunnel.

During the experiment, bees are fixed. And bees body position can be adjusted by the steel wire that fix the bees. The direction of bees body is parallel to the flow direction of wind tunnel.

In the experiment, we can get features pixel point of bees body. The bees head is set as point $\mathrm{A}$ and the bees tail is set as point $\mathrm{B}$. The pixel coordinates on plane $\mathrm{A}$ of point $\mathrm{A}$ and point $\mathrm{B}$ can be used for calculation the throw distance. The throw distance $\mathrm{PaQa}$ can be calculated as follow:

$$
\mathrm{PaQa}=\mathrm{AaBa} \times \frac{\sqrt{(\mathrm{Pa} i-\mathrm{Pa} i)^{2}+(\mathrm{Paj}-\mathrm{Paj})^{2}}}{\sqrt{(\mathrm{Aa} i-\mathrm{Aa} i)^{2}+(\mathrm{Aaj}-\mathrm{Aaj})^{2}}}=\mathrm{AB} \times \frac{\sqrt{(\mathrm{Pa} i-\mathrm{Pa} i)^{2}+(\mathrm{Paj}-\mathrm{Paj})^{2}}}{\sqrt{(\mathrm{Aa} i-\mathrm{Aa} i)^{2}+(\mathrm{Aaj}-\mathrm{Aa} j)^{2}}}(1)
$$

Where projection point on the plane of point $\mathrm{A}$ and point $\mathrm{B}$ is $\mathrm{Aa}$ and $\mathrm{Ba}$. The pixel point coordinates is (Aai, Aaj) and (Bai, Baj). The actual distance from $\mathrm{A}$ to $\mathrm{B}$ is $\mathrm{AB}$. The projector distance on plane $\mathrm{A}$ is $\mathrm{AaBa}$. And The actual distance $\mathrm{AB}$ is $16 \mathrm{~mm}$.

The angle between bees body and horizontal plane is $\delta$. On the plane $B$, projection point of point $A$ and point $B$ is $\mathrm{Ab}$ and $\mathrm{Bb}$ and The projector distance of line $\mathrm{AB}$ on plane $\mathrm{B}$ is $\mathrm{AbBb}$. Where $\mathrm{AbBb}=\mathrm{AB} \times \cos \delta$.

On the plane $\mathrm{B}$, the projection point of point $\mathrm{P}$ and point $\mathrm{Q}$ is $\mathrm{Pb}$ and $\mathrm{Qb}$. The pixel point coordinates of projection point $\mathrm{Ab}, \mathrm{Bb}, \mathrm{Pb}$ and $\mathrm{Qb}$ are (Abi, $\mathrm{Abj}),(\mathrm{Bbi}, \mathrm{Bbj}),(\mathrm{Pbi}, \mathrm{Pbj})$ 
and (Qbi, Qbj). the projector distance on plane A of line PQ is $\mathrm{PbQb}$ :

$$
\mathrm{PbQb}=\mathrm{AbBb} \times \frac{\sqrt{(\mathrm{Pb} i-\mathrm{Pb} i)^{2}+(\mathrm{Pb} j-\mathrm{Pb} j)^{2}}}{\sqrt{(\mathrm{Ab} i-\mathrm{Ab} i)^{2}+(\mathrm{Ab} j-\mathrm{Ab} j)^{2}}}=\mathrm{AB} \times \cos \delta \times \frac{\sqrt{(\mathrm{Pb} i-\mathrm{Pb} i)^{2}+(\mathrm{Pbj} j-\mathrm{Pbj})^{2}}}{\sqrt{(\mathrm{Ab} i-\mathrm{Ab} i)^{2}+(\mathrm{Ab} j-\mathrm{Ab} j)^{2}}}(2)
$$

According to the projection relationship of line and plane, we can get the angle between line PQ and plane A, B. The angle is $\eta_{1}$ and $\eta_{2}$.

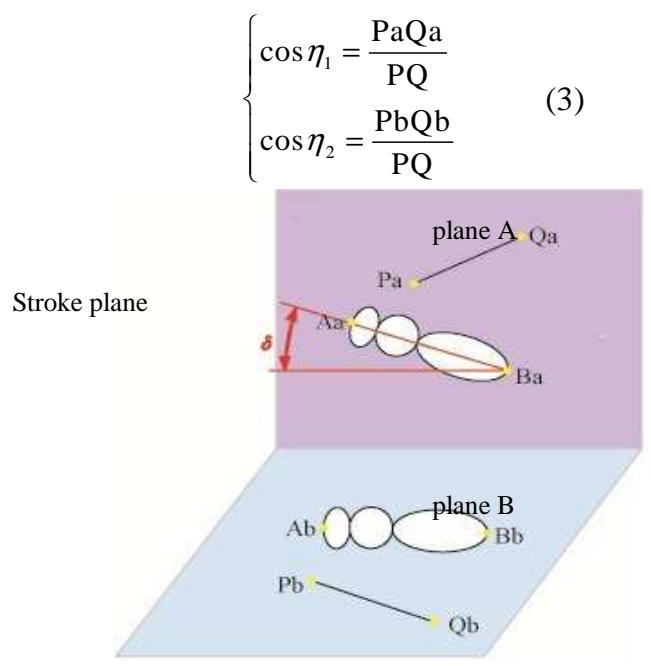

FIGURE II. SCHEMATIC DIAGRAM OF CAMERA PLANE.

\section{The BeE CoORdinAte System}

Because the bees are fixed, the bees flapping motion can be approximately assumed as rigid body rotation around a fixed point. The bees flapping process includes the properties of the rigid motion and the characteristics of the flexible deformation. In the study of the flying of insects and birds, the wings flapping is track is approximately restricted in one plane with the verification of lots of experiment results. The angle that the wings sweep on the stroke plane is called stroke angle. The root point of left wing in the space is defined as point $\mathrm{O}$. Because the bees are fixed, the position of point $A$ remains unchanged. The coordinate system with origin at the root point of bees wing has been built, as shown in Fig. 3. OXYZ is inertial coordinate system. XY plane which is parallel to plane $b$ is horizontal plane. And $\mathrm{Y}$ axis is perpendicular to plane A. Plane $\mathrm{XZ}$ is parallel to the plane A. Oxyz is stroke plane coordinate system for bees. Oxy is stroke plane. The angle between stroke plane and horizontal plane $\mathrm{XY}$ is $\beta$.

The $\mathrm{Y}$ axis coincides with y axis.

The coordinate transformation from coordinate system OXYZ to coordinate system oxyz is shown in formula (4).

$$
\left\{\begin{array}{l}
x=X \cos \beta-Z \sin \beta \\
y=Y \\
z=X \sin \beta+Z \cos \beta
\end{array}\right.
$$

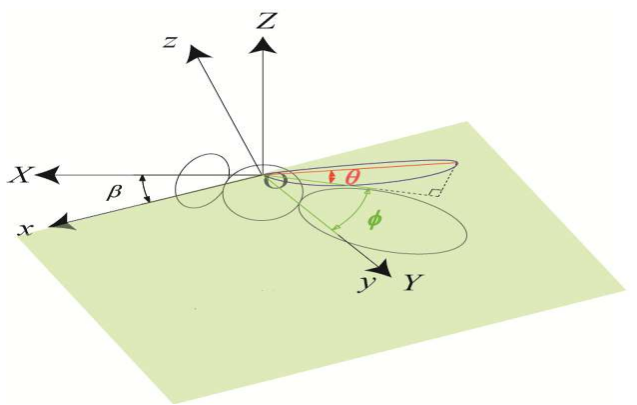

FIGURE III. COORDINATE SYSTEM FOR BEES.

Projection figure of bees on plane OXZ (plane A) is shown in Fig. 4. The body contour of bees is illustrated by red lines. The yellow points represent the projection point of wings tip $\mathrm{C}$ on plane $\mathrm{XZ}$ in one flapping period. The connection of projection point of point $\mathrm{C}$ in one period forms the wing tip track. The blue line is the down-flapping track and the black line is the up-flapping track. The angle between projection on stroke plane and y axis is $\varphi$. The line from the top point of up-flapping Sa to the bottom point $\mathrm{Ra}$ is line A. And the angle between line 1 and horizontal plane is $\beta$. The numerical value of angle $\beta$ can be obtained by pixel point coordinates (Sai, Saj) and pixel point coordinates (Rai, Raj).

$$
\beta=\arctan \left(\left|\frac{\mathrm{Saj}-\mathrm{Ra} j}{\mathrm{Sa} i-\mathrm{Ra} i}\right|\right)
$$

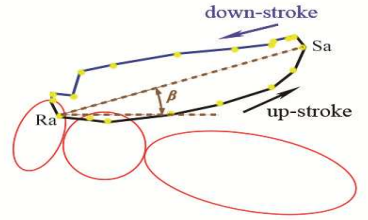

\section{FIGURE IV. SKETCH MAP OF BEES STROKE PLANE ANGLE.}

The bee wing tip is point $\mathrm{C}$. The distance $\mathrm{OC}$ from wing tip point to wing root point is the length of wing. And the length of wing $1=9.8 \mathrm{~mm}$. Wing tip position $C$ changes with bees flapping motion in one flapping period, as well as the pixel point coordinates where point $C$ projects on plane $A$ and plane B. By the analysis of the photo shoot by high speed camera, we can get the pixel point coordinates where point $\mathrm{O}$ and point $\mathrm{C}$ project on plane $\mathrm{OXZ}$ and plane $\mathrm{OXY}$. And we can get the angle $\eta_{\mathrm{cl}}$ between line OC and plane $\mathrm{OXZ}$, as well as the angle $\eta_{\mathrm{c} 2}$ between line $\mathrm{OC}$ and plane OXY. Angle $\eta_{\mathrm{c} 3}$ is assumed as the angle between line OC and plane OXZ. The angle $\eta_{\mathrm{c} 3}$ can be calculated as following:

$$
\sin ^{2} \eta_{\mathrm{c} 1}+\sin ^{2} \eta_{\mathrm{c} 2}+\sin ^{2} \eta_{\mathrm{c} 3}=1
$$

The wing tip point $\mathrm{C}$ on the plane $\mathrm{OXYZ}$ can be get from the length of $\mathrm{OC}$ and angle $\eta_{\mathrm{c} 1}, \eta_{\mathrm{c} 2}, \eta_{\mathrm{c} 3}$.

$$
\left\{\begin{array}{l}
X_{\mathrm{c}}=l \sin \eta_{\mathrm{c} 1} \\
Y_{\mathrm{c}}=l \sin \eta_{\mathrm{c} 2} \\
Z_{\mathrm{c}}=l \sin \eta_{\mathrm{c} 3}
\end{array}\right.
$$


In formula (7), the coordinate value is only algebraic value. Positive or negative of algebraic value should be determined by projection pixel point coordinates of point $\mathrm{C}$.

With formula (4) and (7), coordinate on coordinate system Oxyz of wing tip point $\mathrm{C}$ can be calculated. The angle of amplitude $\Phi$ can be calculated with formula (8):

$$
\tan \phi=-\frac{x_{\mathrm{c}}}{y_{\mathrm{c}}}
$$

The angle $\theta$ between line $\mathrm{OC}$ and stroke plane can be calculated with formula (9):

$$
\tan \theta=\frac{z_{\mathrm{c}}}{\sqrt{x_{c}^{2}+y_{c}^{2}}}
$$

\section{Position Angle And Stroke ANGLE}

Fig. 5 shows the changing curve of stroke angle and position angle in three periods. The variation range of bees position angle is $0 \sim 30^{\circ}$. The stroke angle in one period is approximate sine curve. Fig. 6 shows the stroke angle in one period. And when down-flapping turns into up-flapping in one period, the rotation of wing takes some time. During the time, stroke angle changes few. As shown in Fig.5 and Fig.6, the maximum amplitude of bees wings flapping is about $110^{\circ}$. Compared with $120^{\circ}$ in Ellington's research, $110^{\circ}$ is less because the bees in experiment are fixed.

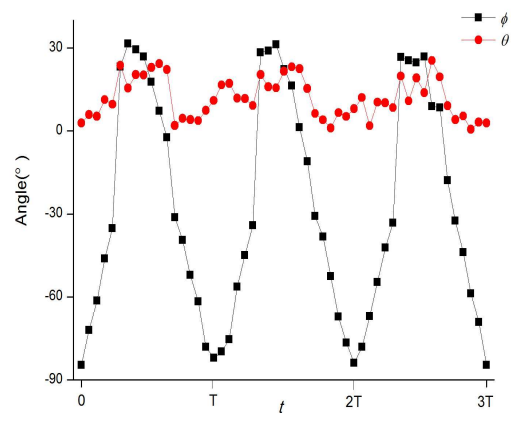

FIGURE V. CURVE GRAPH OF STROKE ANGLE AND POSITION ANGLE IN THREE PERIODS

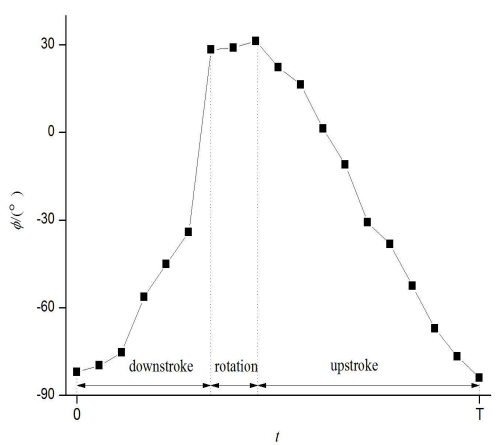

FIGURE VI. CURVE GRAPH OF STROKE ANGLE IN ONE PERIODS.

\section{CONCLUSION}

In this paper, the kinematics coordinate system of bees is established. Two high speed cameras used to record the flapping information synchronously from two different visual angles are applied in the experiment for kinematics regularity of bees flapping. And the bees flapping are periodic. The variation range of position angle is about $30^{\circ}$. Cycle regularity is not obvious. The variation of Stoke angle is approximate sine curve. And the up-flapping time is bigger than down-flapping time. During the metaphase between down-flapping and up flapping, there is one rotation stage. The wings rotate without flapping during the rotation stage.

\section{REFERENCE}

[1] Weis-Fogh, T. \&Jensen, M. Biology and physics of locust flight I.Basic principles in insect flight. A critical review. Philosophical transactions of the Royal Society. 239, pp. 415-458, 1956.

[2] Sane, S. P. and Dickinson, M. H. The control of flight force by a flapping wing: lift and drag production. The journal of experimental biology, 204, pp.2607-2626,2001

[3] Sane, S. P. and Dickinson, M. H. The aerodynamic effects of wing rotation and a revised quasi-steady model of flapping flight. The journal of experimental biology, 205, pp.1087-1096.2002.

[4] Birch,J.M and Dickinson, M. H. The influence of wing-wake interactions on the production of aerodynamic forces in flapping flight The journal of experimental biology, 206, pp.2257-2272, 2003.

[5] Ellington, C. P. The aerodynamics of hovering insect flight.II.Morphological parameters. Philosophical transactions of the Royal Society. 305, pp. 17-40, 1984.

[6] Ellington, C. P. The aerodynamics of hovering insect flight. III.Kinematics. Philosophical transactions of the Royal Society. 305,pp. 41-78,1984 